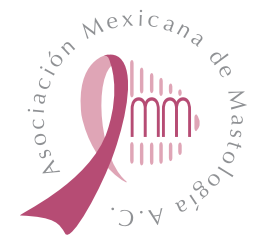

\title{
Cirugía oncoplástica en cáncer de mama: resultados oncológicos con seguimiento en un plazo no menor de 2 años en pacientes
}

\author{
Oncoplastic surgery in breast cancer: oncological results with \\ follow up within a period of no less than 2 years in patients \\ Gerardo Amarante-de León, * Servando Cardo-Huerta, ${ }^{\ddagger}$ Gerardo I Magallanes-Garza, ${ }^{\S}$ \\ Juan C Bermeo-Ortega," Berta Serrano-Peláez," Jorge Santiago-Sánchez**
}

\begin{abstract}
* Especialista en Cirugía
General. Alta Especialidad en Mastología.

‡ Especialista en Cirugía General. Cirugía Oncológica, Director

Nacional de Investigación Clínica Tec Salud.

$\S$ Especialista en Ginecología y

Obstetricia. Alta Especialidad en Mastología. Jefe de Servicio de Ginecología y Obstetricia Tec

Salud.

"Especialista en Cirugía General. Alta Especialidad en Mastología. ** Especialista en Ginecología y Obstetricia. Alta Especialidad en Mastología.
\end{abstract}

Tecnológico de Monterrey. Hospital San José. Centro de Tratamiento de Mama.

Correspondencia: Juan Bermeo-Ortega E-mail: bermeoojc@gmail. com

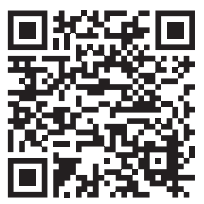

\section{RESUMEN}

Introducción: La cirugía oncoplástica (COP) es un gran avance en cirugía de mama, se establece en dos niveles; el nivel 1 escisión de < del 20\% de volumen mamario y el nivel 2 que abarca técnicas de mamoplastia escisión del 20-50\% del volumen mamario y piel. La COP es una innovación y evolución en el tratamiento del cáncer de mama precoz, brindando un adecuado control oncológico y reflejando un grado de satisfacción alto por las pacientes. Objetivo: Conocer la viabilidad, valorar la respuesta oncológica y el producto estético de las técnicas en cirugía oncoplástica en las pacientes con cáncer de mama con un seguimiento no menor a dos años posterior a la cirugía. Material y métodos: Estudio, descriptivo, retrospectivo, la población incluye a las pacientes intervenidas por cáncer de mama con aplicación de técnicas oncoplásticas. Se utilizó para el análisis estadística descriptiva, basándonos en medidas de tendencia central. Resultados: La media del tamaño tumoral fue $25 \mathrm{~mm}$, el 53.12\%. La cirugía tipo 1 se realizó en 23 casos, 71.87\%. Sólo se registraron dos tipos de complicaciones, hematoma en un caso $3.12 \%$ y necrosis del pezón en dos casos $6.25 \%$. Los márgenes quirúrgicos óptimos $>2 \mathrm{~mm}$ se registraron en 15 casos $46.87 \%$. En cuanto a supervivencia y vigilancia, 31 casos, $96.87 \%$ presentaron remisión completa y se mantienen libres de recurrencia locorregional en un seguimiento no menor de dos años postquirúrgicos. Conclusiones: La COP es una opción el tratamiento primario en pacientes con cáncer de mama en estadios I y II, puesto que permite un control local adecuado, preservando la estética, y no modifica la supervivencia, recurrencias locorregionales ni el índice de metástasis a distancia.

Palabras clave: Cáncer de mama, cirugía oncoplástica, márgenes quirúrgicos, supervivencia, recurrencia.

\section{ABSTRACT}

Introduction: Oncoplastic surgery is a breakthrough in breast surgery, it is established in 2 levels; level 1 excision of < 20\% of breast volume and level 2 encompassing mammoplasty excision techniques of 20-50\% of breast volume and skin. Oncoplastic surgery $(\mathrm{OP})$ is an innovation and evolution in the treatment of the early breast cancer, providing an adequate oncological response control and reflecting a high degree of satisfaction

Citar como: Amarante-de León G, Cardo-Huerta S, Magallanes-Garza GI, Bermeo-Ortega JC, Serrano-Peláez B, Santiago-Sánchez J. Cirugía oncoplástica en cáncer de mama: resultados oncológicos con seguimiento en un plazo no menor de 2 años en pacientes. Rev Mex Mastol. 2020; 10 (1): 6-12. https://dx.doi.org/10.35366/95761 
by the patients. Objective: To know the viability, to evaluate the oncological response and the aesthetic product of the techniques in oncoplastic surgery in patients with breast cancer with a follow-up of at least two years after surgery. Material and methods: A descriptive, retrospective study of the population includes patients undergoing surgery for breast cancer with the application of oncoplastic techniques. The descriptive statistics was used for the analysis, based on measures of central tendency. Results: The mean tumor size was $25 \mathrm{~mm}, 53.12 \%$. Type 1 surgery was performed in 23 cases, $71.87 \%$. Only 2 types of complications were recorded; hematoma in 1 case $3.12 \%$ and necrosis of the nipple in 2 cases $6.25 \%$. The optimal surgical $2 \mathrm{~mm}$ were registered in 15 cases $46.87 \%$. Regarding survival and surveillance; 31 cases $96.87 \%$ presented complete remission and remain free of regional loco recurrence in a follow-up of not less than two years post-surgical. Conclusion: Oncoplastic surgery is an option for primary treatment in patients with stage I and II breast cancer, since it allows adequate local control, preserving aesthetics, and does not modify survival, loco regional recurrences or the rate of distant metastasis.

Keywords: Breast cancer, oncoplastic surgery, surgical margins, survival, recurrence.

\section{INTRODUCCIÓN}

El cáncer de mama es el tumor más frecuente en la mujer, cada año mueren alrededor de 373,000 mujeres, representando el $14 \%$ de todas las muertes por cáncer en el sexo femenino. En Estados Unidos el programa Surveillance Epidemiology and End Results (SEER), analiza los patrones de incidencia, mortalidad y supervivencia en cáncer; concluyendo que el cáncer de mama es la causa más frecuente de muerte en mujeres entre 40 y 55 años con un riesgo acumulado de $8.9 \%$ a lo largo de la vida. ${ }^{1-3}$

La cirugía oncoplástica representa un gran avance en cirugía de mama; la técnica fusiona los principales fundamentos de cirugía oncológica con los procedimientos de la cirugía plástica en el tratamiento conservador del cáncer de mama, favoreciendo el resultado estético final y minimiza el número de cirugías radicales. ${ }^{4-7}$

Numerosas técnicas conforman la cirugía oncoplástica, establecidas en dos niveles. El nivel 1 consiste en técnicas de avance y rotación del tejido glandular, escisión de < del $20 \%$ de volumen mamario no requiere resección de piel, pero sí la centralización del complejo areola-pezón, y el nivel 2 que abarca técnicas de mamoplastia en donde debe ser resecado del $20-50 \%$ del volumen mamario y piel, permitiendo de esta manera la resección de un mayor volumen sin dejar de lado la estética. La COP se basa en tres principios básicos: obtener ediciones amplias, reconstrucción inmediata de la mama e inmediata simetría con la mama contralateral cuando es necesario..$^{8-10}$
Las principales indicaciones en procedimientos oncoplásticos son: resección de $>20 \%$ del volumen mamario, tamaño y localización tumoral, volumen y densidad mamaria, ptosis severa y asimetría, multicentricidad o multifocalidad, macromastia y cirugía plástica previa en la mama. ${ }^{9-12}$

Dentro de las contraindicaciones en cirugía oncoplástica podemos mencionar: tumores T4, cáncer de mama inflamatorio, glándulas mamarias radiadas y enfermedad multicéntrica; cuando no se pueden asegurar márgenes claros sin una mastectomía, poco volumswen mamario y sin ptosis y microcalcificaciones malignas extensas. ${ }^{13-15}$

La cirugía oncoplástica, junto con otras terapias para el control local de la enfermedad oncológica, representa una innovación y evolución en el tratamiento del cáncer de mama precoz, lo cual refleja un grado de satisfacción alto y una disminución de la percepción de la enfermedad por las pacientes. ${ }^{4,16}$

Múltiples son las publicaciones sobre la aplicación de la cirugía oncoplástica en cáncer de mama; sin embargo, hay pocos registros del seguimiento y resultados oncológicos a largo plazo de estas pacientes.

\section{MATERIAL Y MÉTODOS}

Se trata de un estudio observacional, descriptivo, retrospectivo, realizado en el Centro de Tratamiento de Mama del Hospital San José TecSalud, la población incluye a todas las pacientes intervenidas por cáncer de mama en las 
que se aplica técnicas oncoplásticas realizando seguimiento no menor a dos años.

Se seleccionaron los expedientes de los pacientes en los que se encontraba registrado el tipo de intervención oncoplástica realizada y descripción del seguimiento postquirúrgico no menor de dos años. Se excluyeron a las pacientes intervenidas quirúrgicamente en otras instituciones de salud y aquéllas que desistieron del seguimiento postoperatorio. Al obtener los resultados, se tabulan y analizan aplicando técnicas de estadística descriptiva, basados en medidas de tendencia central al utilizar frecuencias en conjunto con porcentajes y la media y mediana en el caso de variables cuantitativas; la significación estadística se estableció como $p<0.05$. Se realizó el análisis de distintas variables como: datos demográficos, parámetros clínicos y quirúrgicos, anatomía patológica, tratamiento realizado y seguimiento.

\section{RESULTADOS}

Datos demográficos

La edad media fue 50.76 (50-59) años y una mediana de 54.5 años, el IMC presentó una media de 27.41 , lo que refleja un predomino del

\begin{tabular}{|c|c|c|}
\hline & $\mathrm{n}$ & $\%$ \\
\hline \multicolumn{3}{|l|}{ Edad } \\
\hline 20-19 & 0 & 0.00 \\
\hline $30-39$ & 2 & 6.00 \\
\hline $40-49$ & 13 & 40.62 \\
\hline $50-59$ & 9 & 28.12 \\
\hline $60-69$ & 7 & 22.00 \\
\hline$>70$ años & 1 & 3.12 \\
\hline \multicolumn{3}{|l|}{ Media: 50.76} \\
\hline \multicolumn{3}{|l|}{ Comorbilidad } \\
\hline HAS & 5 & 15.62 \\
\hline DMT II & 6 & 18.75 \\
\hline Otros & 1 & 3.12 \\
\hline Negados & 20 & 62.50 \\
\hline
\end{tabular}

HAS = hipertensión arterial sistémica; DMT II = diabetes mellitus tipo II.

\begin{tabular}{lrr}
\multicolumn{3}{c}{ Tabla 2: Parámetros clínicos. } \\
\hline Tamaño en mm & $n$ & $\%$ \\
\hline$\leq 20$ & 12 & 37.50 \\
$>\mathbf{2 0}-\leq 50$ & 17 & 53.12 \\
$>\mathbf{5 0}$ & 3 & 9.37 \\
Total & 32 & 100.00 \\
\hline
\end{tabular}

Media $=25 \mathrm{~mm}$.

sobrepeso presente en 13 pacientes (40.62\%). Los antecedentes patológicos mostraron un ligero predominio de la diabetes mellitus tipo II presente en seis pacientes (18.75\%), frente a la hipertensión arterial sistémica presente en cinco pacientes (15.62\%). En cuanto al estado hormonal, se reportaron 18 casos (56.25\%) de pacientes postmenopaúsicas (Tabla 1).

\section{Parámetros clínicos}

El tamaño glandular se estableció mediante la copa del brasier utilizado por las pacientes, con un predominio de la copa B presente en 13 casos (40.62\%). La densidad mamaria obtenida a través de mamografía demostró un patrón $\mathrm{C}$ en 15 casos (46.87\%). En cuanto a la lateralidad tumoral en la glándula mamaria, se identificaron 19 casos (59.37\%) que afectan al lado izquierdo, no se reportaron casos de bilateralidad. Para determinar el tamaño tumoral, invasión ganglionar y la etapa clínica de la enfermedad, se utilizaron los sistemas de clasificación internacional de la American Joint Committee on Cancer (AJCC) y estadificación TNM. El tamaño tumoral reflejó una media de $25 \mathrm{~mm}$ (> $20-\leq 50 \mathrm{~mm}$ ), presentando 17 casos (53.12\%) y que corresponden según la estadificación TNM al grupo T2 para tamaño tumoral. Se encontró una diferencia estadísticamente significativa $(p=0.01)$ entre el tamaño tumoral determinado clínicamente y el tamaño establecido por patología, no fue así el caso de la relación entre el tamaño tumoral determinado clínicamente y aquel que se estableció mediante estudios de imagen, específicamente a través de ultrasonido, en donde no hubo una diferencia estadística significativa $(p$ $=0.44$ ). En cuanto a la presencia de ganglios 


\begin{tabular}{|c|c|c|}
\hline & $\mathrm{n}$ & $\%$ \\
\hline \multicolumn{3}{|l|}{ Tipo de cirugía } \\
\hline Tipo 1 & 23 & 71.87 \\
\hline Tipo 2 & 9 & 28.12 \\
\hline \multicolumn{3}{|l|}{ Complicaciones } \\
\hline Hematoma & 1 & 3.12 \\
\hline $\begin{array}{l}\text { Necrosis del } \\
\text { pezón }\end{array}$ & 2 & 6.25 \\
\hline Ninguna & 29 & 90.62 \\
\hline \multicolumn{3}{|l|}{ Reintervenciones } \\
\hline Sí & 1 & 3.12 \\
\hline No & 31 & 96.87 \\
\hline
\end{tabular}

linfáticos, se presentaron 25 casos (78.12\%) que corresponden a un estado N0. La etapa clínica del cáncer de mama II A se presentó en 17 casos (53.12\%) (Tabla 2).

\section{Parámetros quirúrgicos}

Fueron intervenidas 32 pacientes con diagnóstico de cáncer de mama y que eran candidatas a técnicas de cirugía oncoplástica. Se identificaron 23 casos (71.87\%) en los cuales se realizó cirugía tipo 1. Las complicaciones postquirúrgicas estuvieron ausentes en 29 casos $(90.62 \%)$, sólo se registraron dos tipos de complicaciones: hematoma presente en un caso $(3.12 \%)$ y necrosis del pezón presente en dos casos (6.25\%) (Tabla 3).

\section{Parámetros de anatomía patológica}

Como se mencionó con anterioridad, dentro de los parámetros clínicos y en relación al tamaño tumoral, se encontró una diferencia estadísticamente significativa $(p=0.01)$ entre el tamaño tumoral determinado clínicamente y el tamaño establecido por patología. El subtipo molecular de cáncer de mama que predominó fue el luminal, en donde se reportaron 25 casos (78\%), seguido del subtipo triple negativo con cuatro casos (12.5\%). El grado histológico de diferenciación presentó una media de 10.66, el grado 2 estuvo presente en 13 casos 40.62\%, con un rango similar se presentó el grado 3 con 12 casos (37.5\%). El tipo histológico más frecuente fue el carcinoma ductal infiltrante (CDI) con 26 casos (82\%), seguido del carcinoma lobulillar infiltrante (CLI) y del carcinoma ductal in situ (CDIS) con tres casos cada uno. Los márgenes quirúrgicos óptimos $>2 \mathrm{~mm}$ se registraron en 15 casos (46.87\%). Sólo en cinco casos (15.62\%) los márgenes quirúrgicos no pudieron valorarse debido a ausencia del tumor por biopsia escisional previa o por respuesta patológica completa (Tabla 4).

\section{Tratamientos}

El tratamiento sistémico se administró según lo indicado en el consenso multidisciplinario en cada caso (Tabla 5).

No se encontró una diferencia estadísticamente significativa $(p=0.13)$ en relación con el tamaño tumoral entre los pacientes que recibieron quimioterapia neoadyuvante y los que no lo hicieron. El estado ganglionar

\begin{tabular}{|c|c|c|}
\hline \multicolumn{3}{|c|}{$\begin{array}{l}\text { Tabla 4: Parámetros de } \\
\text { anatomía patológica. }\end{array}$} \\
\hline & $\mathrm{n}$ & $\%$ \\
\hline \multicolumn{3}{|l|}{$\begin{array}{l}\text { Subtipo molecular cáncer de } \\
\text { mama COP }\end{array}$} \\
\hline Luminal & 25 & 78.00 \\
\hline Her2 + & 3 & 9.37 \\
\hline Triple negativo & 4 & 12.50 \\
\hline \multicolumn{3}{|l|}{ Grado histológico } \\
\hline 1 & 7 & 21.87 \\
\hline 2 & 13 & 40.62 \\
\hline 3 & 12 & 37.50 \\
\hline \multicolumn{3}{|l|}{ Media: 10.66, mediana: 12} \\
\hline \multicolumn{3}{|l|}{ Histología tumoral } \\
\hline $\begin{array}{l}\text { Carcinoma ductal infiltrante } \\
\text { (CDI) }\end{array}$ & 26 & 82.00 \\
\hline $\begin{array}{l}\text { Carcinoma ductal in situ } \\
\text { (CDIS) }\end{array}$ & 3 & 9.00 \\
\hline $\begin{array}{l}\text { Carcinoma lobulillar } \\
\text { infiltrante (CLI) }\end{array}$ & 3 & 9.00 \\
\hline \multicolumn{3}{|l|}{ Márgenes quirúrgicos } \\
\hline $1-2 \mathrm{~mm}$ & 12 & 37.50 \\
\hline$>2 \mathrm{~mm}$ & 15 & 46.87 \\
\hline No valorable & 5 & 15.62 \\
\hline
\end{tabular}


linfático tampoco demostró una diferencia significativa $(p=0.21)$ entre pacientes sometidos a quimioterapia neoadyuvante y los que no fueron sometidos a esta terapéutica inicial. De igual manera, al referirnos a la etapa clínica en relación con pacientes que recibieron quimioterapia neoadyuvante, no se encontró una diferencia significativa $(p=0.09)$ (Tabla 6).

La radioterapia posterior a cirugía se administró en todas las pacientes que intervinieron en este estudio (32 casos, 100\%). El tratamiento hormonal a base de tamoxifeno se estableció en nueve casos $(28.12 \%)$, mientras que los inhibidores de aromatasa se utilizaron en 15 casos (46.87\%).

Vigilancia y sobrevida

De las 32 pacientes que intervinieron en este estudio, 31 casos $(96.87 \%)$ presentaron remisión completa y se mantienen libres de recurrencia locorregional en un seguimiento no menor de dos años postquirúrgicos, se encontró un caso (3\%) con hallazgos a dos años tres meses después de la cirugía; reporte de mamografía/ultrasonido: categoría BI-RADS 4b, zona de distorsión con moderada sospecha de malignidad, se realiza biopsia guiada con arpón y el reporte de patología indica una hiperplasia ductal de tipo usual. De igual manera, resulta importante señalar que se presentó un caso de fallecimiento no relacionado con cáncer de mama (Tabla 7).

\section{DISCUSIÓN}

La cirugía oncoplástica representa un gran avance en cirugía de mama, la técnica fusiona los principales fundamentos de cirugía oncológica con los procedimientos de la cirugía plástica en el tratamiento conservador del cáncer

Tabla 5: Quimioterapia neoadyuvante.

\begin{tabular}{lrr} 
& $n$ & \multicolumn{1}{c}{$\%$} \\
\hline Sí & 7 & 21.87 \\
No & 25 & 78.12 \\
Total & 32 & 100.00 \\
\hline
\end{tabular}

\begin{tabular}{lrrr}
\multicolumn{4}{c}{$\begin{array}{c}\text { Tabla 6: Relación quimioterapia } \\
\text { neoadyuvante con tamaño } \\
\text { tumoral y etapa clínica. }\end{array}$} \\
Sí & No & p \\
\hline & 7 & 25 & \\
\hline Quimioterapia neoadyuvante & & & 0.13 \\
Tamaño tumoral & 0 & 0 & \\
T0 & 0 & 10 & \\
T1 & 5 & 14 & \\
T2 & 2 & 1 & \\
T3 & & & 0.21 \\
Estado ganglionar & 3 & 22 & \\
N0 & 2 & 3 & \\
N1 & 2 & 0 & \\
N2 & & & 0.09 \\
Etapa clínica & 0 & 8 & \\
I & 3 & 14 & \\
IIA & 1 & 2 & \\
IIB & 3 & 1 & \\
IIIA & & & \\
\hline
\end{tabular}

de mama, favoreciendo el resultado estético final y minimiza el número de cirugías radicales.

El presente trabajo, al ser un estudio retrospectivo, tiene limitaciones relativas al trabajar con un número de casos reducidos; sin embargo, al analizar los resultados obtenidos en nuestro estudio y al compararlos con publicaciones previas, se relacionan en gran medida.

Dentro de los datos demográficos es importante resaltar que factores como la edad media, predominio de comorbilidades como hipertensión arterial y diabetes mellitus fueron similares a los reportados en otros estudios; Brito G y Sherwell S y colaboradores ${ }^{1,2}$ establecen una edad media de 54.2 años, una prevalencia de la hipertensión arterial 14.5\% en relación con los resultados obtenidos en nuestro estudio, en donde se evidencia una edad media de 50.76 años y un porcentaje del $15.62 \%$ para la hipertensión arterial. El tamaño tumoral reflejo una media de $25 \mathrm{~mm}(>20$ $\leq 50 \mathrm{~mm}$ ), presentando 17 casos (53.12\%) y que corresponden según la estadificación TNM al grupo T2, valores que se asemejan a lo reportado por Hechavarría Y y su equipo, ${ }^{5}$ quienes en su investigación indican que el $62 \%$ de los tumores correspondían a una estadificación TNM T2. 


\begin{tabular}{lrr}
\hline \multicolumn{3}{c}{ Tabla 7: Progresión } \\
locorregional y metástasis. \\
\hline \\
& $\mathrm{n}$ & $\%$ \\
\hline Libre recurrencia locorregional & 31.00 & 96.87 \\
Mets & 0.00 & 0.00 \\
Otros hallazgos & 1.00 & 3.00 \\
Fallecidas & 0.00 & 0.00 \\
Total & 32.00 & 100.00 \\
\hline
\end{tabular}

Se identificaron 23 casos (71.87\%) en los cuales se realizó cirugía oncoplástica tipo 1 y la cirugía oncoplástica tipo 2 se realizó en nueve casos $(28.12 \%)$, dichos resultados se asemejan a lo publicado por Garcés $M$ y su grupo de investigadores $^{6}$ en su experiencia en el Departamento de Cirugía en Mama del Instituto de Enfermedades Neoplásicas de Lima quien reportó un $65.7 \%$ de pacientes que fueron sometidas a cirugía oncoplástica tipo 1, mientras que la cirugía tipo 2 fue reportada en un 34.9\% de los casos.

El tipo histológico más frecuente fue el carcinoma ductal infiltrante (CDI) $82 \%$, Acea Nebril B y colaboradores ${ }^{11}$ reportaron un $73.2 \%$ con histología de carcinoma ductal infiltrante, lo cual se asemeja a lo descrito en el presente estudio.

Se indicó quimioterapia neoadyuvante en el $21.87 \%$ de los casos, mientras que en el $78.12 \%$ no la recibieron. No se encontró una diferencia estadísticamente significativa $(p=0.13)$ en relación al tamaño tumoral entre los pacientes que recibieron quimioterapia neoadyuvante y los que no lo hicieron. El estado ganglionar linfático tampoco demostró una diferencia significativa $(p=0.21)$ entre pacientes sometidos a quimioterapia neoadyuvante y los que no la recibieron. Hernando $E^{16}$ reportó en su estudio un $10.3 \%$ de casos que recibieron quimioterapia neoadyuvante y un $89.7 \%$ que no la recibieron; además expresa que no hay una diferencia significativa $(p=0.70)$ en relación al tamaño tumoral y estado ganglionar $(p=0.28)$ , resultados que se asemejan a lo descrito por el presente estudio.

De las 32 pacientes que participaron en esta investigación, 31 casos (96.87\%) presentaron remisión completa y se mantienen libres de recurrencia locorregional en un seguimiento no menor de dos años postquirúrgicos. Sherwell S y Cháveza M y colaboradores ${ }^{2,8}$ indican que se han reportado en estudios similares una tasa de recurrencia posterior a cirugía oncoplástica entre el 2 y $7 \%$, no obstante, en sus publicaciones no se han presentado recurrencias locales, pero recomiendan un periodo de seguimiento a mayor plazo.

\section{CONCLUSIONES}

La cirugía oncoplástica es una opción de tratamiento primario en pacientes con cáncer de mama en estadios I y II, puesto que permite un control local adecuado, preservando la estética y no modifica la supervivencia, recurrencias locorregionales, ni el índice de metástasis a distancia. Evidenciando un grado alto en cuanto a la satisfacción emocional y estética de las pacientes que son intervenidas quirúrgicamente por este tipo de alternativa quirúrgica.

\section{BIBLIOGRAFÍA}

1. Brito SG, Pérez BIM. Conservative surgery for breast cancer. Revista Cubana de Cirugía. 2014; 53 (2): 201212.

2. Sherwell-Cabello S, Maffuz-Aziz A, Villegas-Carlos F, Domínguez-Reyes C, Labastida-Almendaro S, Rodríguez-Cuevas S. Feasibility and cosmetic outcome of oncoplastic surgery in breast cancer treatment. Cir Cir. 2015; 83 (3): 199-205.

3. Martínez Gómez E, Cano Cuetos A, Medina Garrido C, Canseco Martín C, Arnanz Velasco F, Garrido Sánchez $\mathrm{N}$ et al. Cáncer de mama en mujeres muy jóvenes, nuestra experiencia. Clin Invest Gin Obst. 2016; 43 (1): 17-23.

4. De Miranda Pires D, Gazoto Junior O, Nazareth Valadares C, Levy Andrade R. Formação em cirurgia oncoplástica e reconstrutiva da mama: análise da formação na américa e na união europeia com a realidade brasileira. Mastology. 2017; 27 (2): 164-71.

5. Hechavarría JY, Ramírez JR, Fernández SP, Portelles CA, Cedeño MA Oncoplastic surgery for breast cancer in patients attended at "Vladimir Ilich Lenin" Hospital. Correo Científico Médico. 2015; 19 (4): 1-13.

6. Garcés M, Mendoza G, Falla M, Cotrina J, Sarria G, Doimi F et al. Oncoplastic surgery of the breast: First experience at the Instituto Nacional de Enfermedades Neoplasicas. Carcinos. 2013; 3 (2): 3-11.

7. Cruz Benítez L, Morales Hernández ER. Historia y estado actual sobre los tipos de procedimientos quirúrgicos realizados en cáncer de mama. Gamo. 2014; 13 (2): 124-133. 
8. Chávez M, Ziegler G, Cotrina M, Garcés M, Calderón G, Mantilla R. Resultados oncológicos de la cirugía oncoplástica: seguimiento a largo plazo de pacientes tratados en un instituto oncológico de Latinoamérica. Rev Senol Patol Mamar. 2017; 30 (4): 170-178.

9. Villarreal CSP. La cirugía oncoplástica en cáncer de mama. Rev Mex Mastol. 2014; 4 (2): 45-51.

10. Mathelin C, Dissaux C, Ruffenach L, Bruant-Rodier C. Cirugía oncoplástica de la mama. EMC - Cirugía Plástica Reparadora y Estética. 2018; 2 6(1).

11. Acea Nebril B, Cereijo Garea C, García Novo A. Cosmetic sequelae after oncoplastic surgery of the breast. Classification and factors for prevention. Cir Esp. 2015; 93 (2): 75-83.

12. Escandón EYM, Zepeda CEJ, Ibarra PÁS. Complicaciones de la reconstrucción mamaria en pacientes oncológicas del Hospital Regional $1^{\circ}$ de Octubre del ISSSTE. Rev Esp Med Quir. 2013; 18 (2): 131-137.

13. Vázquez Albadalejo C. Cirugía del cáncer de mama: técnicas quirúrgicas de tratamiento y de reconstrucción, momento y tiempos de recuperación. Med Segur Trab. 2016; 62 (Suppl): 116-124.

14. Garcés M, Falla M, Mendoza Z, Cotrina J, Ruíz M. La cirugía oncoplástica de la mama: Una técnica quirúrgica que mejora la calidad de vida de las pacientes. Rev Med Hered. 2016; 27 (4): 256-263.

15. Garces M, Mendoza G, Falla M, Ruiz M. Oncoplastic breast surgery: Peruvian experience in a public hospital. Carcinos. 2015; 5 (2): 39-46.

16. Hernando E, Casamayor MC, Ibáñez R, Palomares A, Rodrigo V, Cruz S et al. Oncoplastic surgery and intraoperative radiotherapy in breast cancer. Rev Senol Patol Mamar. 2019; 32 (1): 12-16. 